\title{
Mobility-shift analysis with microfluidics chips
}

\author{
Jarrod Clark ${ }^{1}$, Taras Shevchuk ${ }^{1}$, Piotr M. Swiderski ${ }^{1}$, Rajesh Dabur ${ }^{1}$, Laura E. Crocitto ${ }^{1}$, \\ Yaroslav I. Buryanov ${ }^{2}$, and Steven S. Smith ${ }^{1}$
}

BioTechniques 35:548-554 (September 2003)

\begin{abstract}
Electrophoretic mobility shift analysis (EMSA) is a well-characterized and widely used technique for the analysis of protein-DNA interaction and the analysis of transcription factor combinatorics. Currently implemented EMSA generally involves the time-consuming use of radiolabeled DNA and polyacrylamide gel electrophoresis. We are studying the bionanoscience of self-assembling supramolecular protein-nucleic nanostructures. We have undertaken these studies because they promise to enhance our understanding of assemblies formed during prebiotic evolution, provide tools for analysis of biological processes like DNA recombination, and may lead to the development of nanoscale biosensors designed for site-specific molecular targeting. During the course of that work, we noted that EMSA of these complex structures could be effectively implemented with microfluidics chips designed for the separation of DNA fragments. In this report we compare the two techniques and demonstrate that the microfluidics system is also capable of resolving complex mixtures produced by decorating DNA recombination intermediates with mixtures of DNA binding proteins. Moreover, the microfluidics chip system improves EMSA by permitting analysis with smaller samples, avoiding the use of radiolabeling, and reducing the time involved to a matter of minutes.
\end{abstract}

\section{INTRODUCTION}

Electrophoretic mobility shift analysis (EMSA) is widely used in the analysis of protein-DNA interaction and transcription factor combinatorics (1-5). Interaction with plasmid DNAs, viral DNAs, and oligodeoxynucleotides containing single and multiple binding sites have been studied. In the oligodeoxynucleotide implementation of the method, partially purified DNA binding proteins are mixed with radiolabeled (3) or fluorescent (6) oligodeoxynucleotides in a binding buffer. Subsequent analysis involves agarose or polyacrylamide gel electrophoresis gel drying and fluorescence photography or autoradiography. While this has been an extraordinarily useful and productive technique, it is time-consuming. Moreover, the necessity for radioactive materials (generally ${ }^{32} \mathrm{P}$ ) in many implementations is undesirable.

We are studying the bionanoscience of self-assembling supramolecular protein-nucleic nanostructures. We have undertaken these studies because they promise to enhance our understanding of assemblies formed during prebiotic evolution, provide tools for analysis of biological processes like DNA recombination, and may lead to the development of nanoscale biosensors designed for site-specific molecular targeting. Achieving these goals will involve the construction of several types of biosensors and the development of programmable devices $(7,8)$ with the complexity of prebiotic metabolosomes (9) or even small viruses. In order to make progress toward these goals, enabling tools for the rapid structural analysis of these systems must be developed. One such tool is EMSA. Recently, we noted that microfluidics chips designed for the separation of duplex DNA fragments could be employed in EMSA (http://www.foresight.org/ Conferences/MNT10/Abstracts/Clark/ index.html). In this report, we briefly describe the method and its utility.

\section{MATERIALS AND METHODS}

\section{Enzymes and Chemicals}

Methylase HhaI $(\mathrm{M} \cdot H h a \mathrm{I})$ was purified as previously described (10).
Cloning of the methylase EcoRII nuclear localization signal $(\mathrm{M} \bullet E c o$ RIINLS) fusion protein was performed using standard recombinant DNA techniques. Purification of this fusion protein was carried out using a modification of the procedure described previously $(11,12)$. The procedure is representative of those employed in this work and can be summarized as follows. First Escherichia coli carrying the M•EcoRII-NLS fusion protein on plasmid pRT103 were grown to stationary state, harvested by centrifugation at $5000 \times g$ for $10 \mathrm{~min}$ and resuspended in lysis buffer at a density of $0.67 \mathrm{~g}$ cells $/ \mathrm{mL}$ to break down the bacterial cell walls causing the cells to lyse. The lysis buffer contained $50 \mathrm{mM}$ Tris-HCl, $\mathrm{pH} 7.8,10 \%$ v/v glycerol, 1 mM EDTA, and $750 \mu \mathrm{g} / \mathrm{mL}$ lysozyme (Sigma, St. Louis, MO, USA). After 30 min at $0^{\circ} \mathrm{C}$, the mixture was incubated for $5 \mathrm{~min}$ at $20^{\circ} \mathrm{C}$. The viscous mixture was sonicated five times in 15-s bursts, with 1 min cooling at $0^{\circ} \mathrm{C}$ between bursts at the microtip maximum setting with a Model 450 Digital Sonifier (Branson Ultrasonics, Danbury, CT, USA). This step stimulates lysis

\footnotetext{
${ }^{1}$ City of Hope Medical Center, Duarte, CA, USA and ${ }^{2}$ Russian Academy of Sciences, Moscow, Russia
} 
Table 1. Oligonucleotides Used in This Study

\begin{tabular}{|c|c|}
\hline Oligodeoxynucleotide & Sequence \\
\hline M.Hha I duplex top & $5^{\prime}-$ TCACCAGATGCGCTGTAGGTCGTGTGCGCTGGTTCCACCAGAGCGCTGTGACCTGTAGTT-3' \\
\hline M.Hha I duplex bottom & $5^{\prime}$-AACTACAGGTCACAGCGCTCTGGTGGAACCAGCGCACACGACCTACAGCGCATCTGGTGA-3'$\stackrel{\mathrm{M}}{\mathrm{M}}$ \\
\hline $\begin{array}{c}\text { Y-Junction Arm One } \\
\text { (M·Hha I or M·Eco RII) }\end{array}$ & $5^{\prime}$ - TCACCAGATTGTAGCGCGGTCGTGCTACCTGGTTCCACCAGATGCGCGTGACCTGTAGTT-3' \\
\hline $\begin{array}{c}\text { M.Hha I Y-Junction } \\
\text { Arm Two }\end{array}$ & 5' -AACTACAGGTCACGCGCATCTGGTGGAACCCAGTTAGCCTCAAGCGCTGGCATAGCCAGC-3' \\
\hline $\begin{array}{c}\text { M·Hha I Y-junction } \\
\text { Arm Three }\end{array}$ & 5' - -GCTGGCTATGCCAGCGCTTGAGGCTAACTGAGGTAGCACGACCGCGCTACAATCTGGTGA-3' \\
\hline $\begin{array}{c}\text { M.Eco RII Y-Junction } \\
\text { Arm Two }\end{array}$ & $5^{\prime}$ - -AACTACAGGTCACGCGCATCTGGTGGAACCCAGTTAGCCTCACCTGGTGGCATAGCCAGC-3 \\
\hline $\begin{array}{c}\text { M·Eco RII Y-Junction } \\
\text { Arm Three }\end{array}$ & 5' -GCTGGCTATGCCACCAGGTGAGGCTAACTGAGGTAGCACGACCGCGCTACAATCTGGTGA-3' \\
\hline \multicolumn{2}{|c|}{$\left(\begin{array}{l}M \\
C\end{array}\right)$ indicates position of 5-methylcytosine and $\left(\begin{array}{l}F \\
C\end{array}\right)$ indicates position of 5-fluorocytosine } \\
\hline
\end{tabular}

of any intact cells and fragments the DNA released during lysis, thereby reducing the viscosity of the solution. Temperature during sonication was maintained below $15^{\circ} \mathrm{C}$. Cellular debris was removed by centrifugation at $5000 \times g$ for $10 \mathrm{~min}$. To remove nucleic acid and partially fractionate the protein mixture, the supernatant fluid was then mixed with an equal volume of diethylaminoethyl (DEAE) Sepharose ${ }^{\mathrm{TM}}$ (Amersham Biosciences, Uppsala, Sweden) that had been equilibrated with a buffer containing $50 \mathrm{mM}$ Tris-HCl, pH 7.8, 10\% v/v glycerol, 1 mM EDTA, and $250 \mathrm{mM} \mathrm{NaCl}$. After stirring for $30 \mathrm{~min}$, the unbound material was collected by filtration.

The filtrate was then mixed with one-half volume of precycled phosphocelluolse (P-11; Whatman, Maidstone, $\mathrm{UK}$ ), to recover nucleic acid binding proteins. After stirring for $30 \mathrm{~min}$, the mixture was filtered, and the P-11 cake was resuspended in 2 volumes of the same buffer containing $150 \mathrm{mM} \mathrm{NaCl}$. The cake was again collected by filtration and resuspended in 2 volumes of the same buffer containing $400 \mathrm{mM}$ $\mathrm{NaCl}$. Elution was carried out by filtration. Further purification of the meth- yltransferase was achieved by applying the eluted proteins (approximately 170 $\mathrm{mg}$ ) to two tandemly linked 5-mL Cibacron-blue Econo-Pac ${ }^{\circledR}$ fast-protein liquid chromatography (FPLC) columns (Bio-Rad Laboratories, Hercules, CA, USA). After washing the columns with $50 \mathrm{~mL}$ of the same buffer containing $180 \mathrm{mM}$ salt, elution was carried out with a step increase in salt concentration to $1.5 \mathrm{M} \mathrm{NaCl}$ in the same buffer. In this final purification step, gradients were prepared and protein was detected with a Millennium ${ }^{32}{ }^{\circledR}$ System (Waters, Milford, MA, USA). Active fractions were then concentrated using Amicon ${ }^{\circledR}$ Centricon ${ }^{\circledR}-10$ concentrators (Millipore, Billerica, MA, USA). Unlike the $\mathrm{M} \bullet H h a \mathrm{I}$ preparation, which was essentially homogeneous by polyacrylamide gel electrophoresis, the M•EcoRII-NLS fusion preparation contained approximately 15 visible bands on polyacrylamide gels stained with Coomassie ${ }^{\circledR}$ Blue R250 (Sigma), suggesting that it made up about $20 \%$ of the visible protein.

\section{Oligodeoxynucleotide Preparation}

Oligodeoxynucleotides were synthe- sized by the City of Hope DNA Synthesis facility using standard phosphoramidite chemistry. 5Fluorodeoxycytidine was introduced as the TMP-F-dU-CE convertible phosphoramidite (Glen Research, Sterling, VA, USA). Concentrations were measured by absorbance spectroscopy at 260 $\mathrm{nm}$. To form duplexes and Y-junctions, oligodeoxynucleotides were mixed in equimolar amounts in a buffer containing $10 \mathrm{mM}$ Tris$\mathrm{HCl}, \mathrm{pH} 7.2,1 \mathrm{mM}$ EDTA, and $100 \mathrm{mM} \mathrm{NaCl}$, to a final concentration of $6 \mu \mathrm{M}$. They were then annealed at $95^{\circ} \mathrm{C}$ for $5 \mathrm{~min}, 50^{\circ} \mathrm{C}$ for $60 \mathrm{~min}$, room temperature for $10 \mathrm{~min}$, and then put on ice for $10 \mathrm{~min}$. Oligodeoxynucleotide sequences used in this study are listed in Table 1.

\section{Protein Binding Conditions}

Annealed Y-junctions, at concentrations ranging from 0.37 to $1.5 \mu \mathrm{M}$, were exposed to concentrations of $\mathrm{M} \cdot H h a \mathrm{I}$ ranging from 0.81 to $2.43 \mu \mathrm{M}$ in a binding buffer containing $50 \mathrm{mM}$ Tris- $\mathrm{HCl}, \mathrm{pH} 7.8$, $10 \mathrm{mM}$ EDTA, $5 \mathrm{mM} \beta$-mercaptoethanol, and $80 \mu \mathrm{M}$ S-adenosyl-L-methionine (AdoMet). The final volume of the reaction was $10 \mu \mathrm{L}$. The reaction was then incubated for $2.5 \mathrm{~h}$ at $37^{\circ} \mathrm{C}$. When the $\mathrm{M} \bullet E c o$ RII-NLS fusion protein was employed, it was mixed with $0.6 \mu \mathrm{M}$ Y-junction under the same conditions as in a $10-\mu \mathrm{L}$ reaction volume. In this case, $6.3 \mu \mathrm{g} / \mathrm{mL}$ of partially purified protein was used.

\section{Microfluidics Separations}

The DNA 500 LabChip ${ }^{\circledR}$ (Agilent, Palo Alto, CA, USA) was used for separation of oligodeoxynucleotides, DNA duplexes, frayed DNA duplexes, and the DNA Y-junction, since it gave good resolution in this low mobility range. The DNA 7500 LabChip (Agilent) was used for the detection of retardation products, since the higher occupancy products had mobilities that were outside the separation range of the DNA 500 LabChip. Prior to separation, $9 \mu \mathrm{L}$ of gel-dye mixture were added to the appropriate well, 
and the chip was primed with $0.8 \mathrm{~mL}$ of air for $30 \mathrm{~s}$ using the Agilent priming station. Each of the two gel reservoir wells was then loaded with $9 \mu \mathrm{L}$ of gel-dye mixture. When the DNA 500 LabChip was used, $5 \mu \mathrm{L}$ of the 500 gel-dye mixture were added to each well, and $1 \mu \mathrm{L}$ of the 500-chip ladder was added to the ladder well. When the DNA 7500 LabChip was used, $5 \mu \mathrm{L}$ of the 7500 gel-dye mixture, $5 \mu \mathrm{L}$ of the internal marker mixture, and $1 \mu \mathrm{L}$ of the 7500-chip ladder were added to the ladder well. One microliter from each reaction was added to one of the twelve sample wells. The chip was then vortex mixed at $2400 \mathrm{rpm}$ for $1 \mathrm{~min}$ and then placed in the Bioanalyzer 2100 (Agilent) within $5 \mathrm{~min}$.

\section{Polyacrylamide Gel Electrophoresis}

EMSA using standard polyacrylamide gel electrophoresis was carried

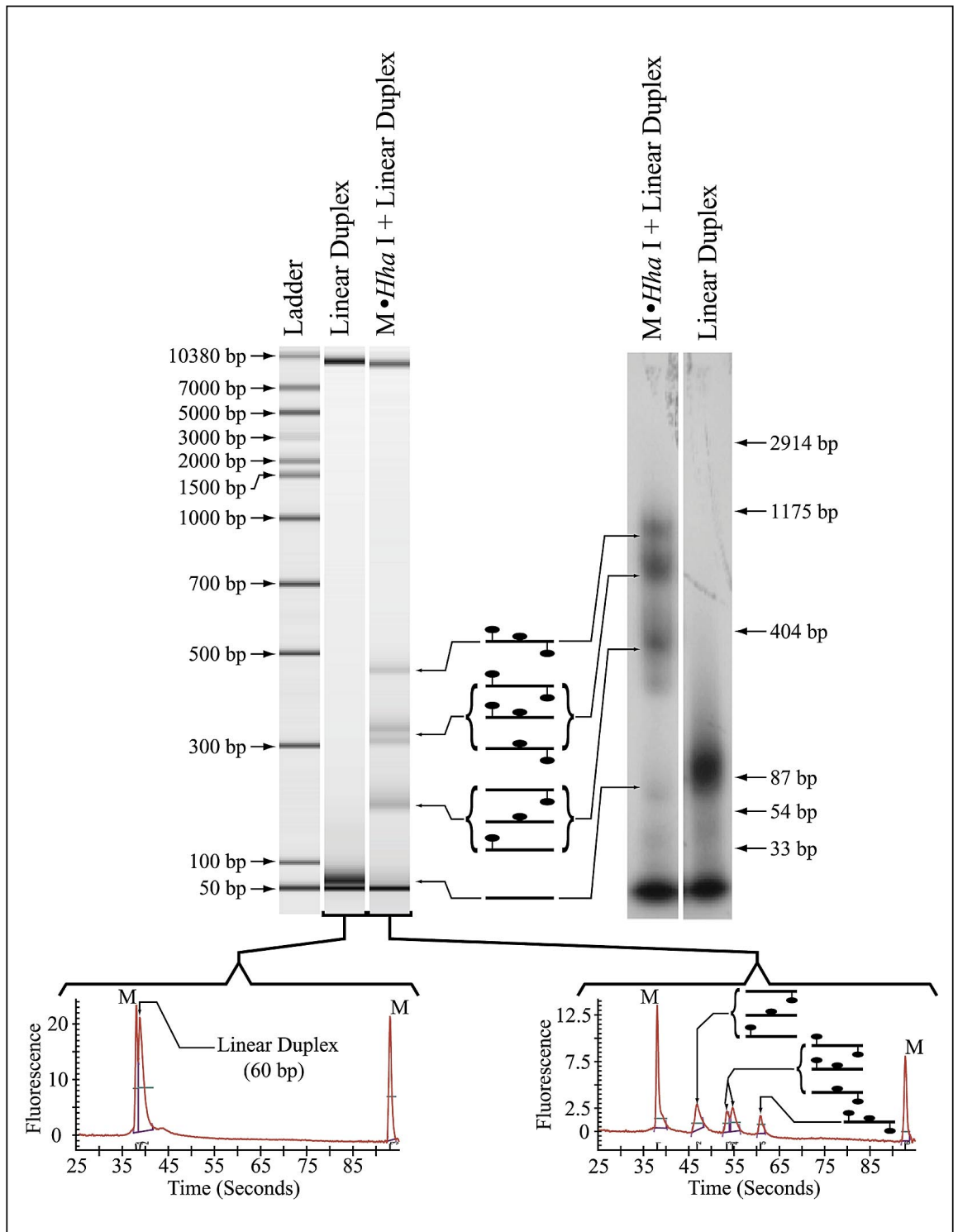

Figure 1. Comparison of microfluidics and gel electrophoretic methodology. In this experiment, a linear duplex DNA is decorated with three copies of methylase $H h a \mathrm{I}(\mathrm{M} \bullet H h a \mathrm{I})$. Recognition sequences were placed at distances that permit the formation of a self-assembling nano-camshaft. The microfluidics separation is presented as a vitural gel on the left, the autoradiograph of the polyacrylamide gel separation is represented on the right. Microfluidics displays of the scanned peaks are presented at the bottom of the figure. Stick figure representations of the undecorated duplex or of the duplex decorated with one, two, or three copies of $\mathrm{M} \bullet \mathrm{HhaI}$ are given in the center of the diagram and in the inset scan in the lower right. Lanes marked Ladder contain the internal markers used in the microfluidics chip. out by loading $32-\mu \mathrm{L}$ samples onto an $11-\mathrm{cm}$ linear $6 \%-20 \%$ acrylamide gradient gel and run at $8.0 \mathrm{~V} / \mathrm{cm}$ and $25 \mathrm{~mA}$ for $3 \mathrm{~h}$. TaqI-digested ${ }^{32} \mathrm{P}$-endlabeled ФX174RFI (New England Biolabs, Beverly, MA, USA) provided molecular weight markers. The gel was then directly exposed to X-ray film for autoradiography $(10,13)$. Autoradiographs were scanned and analyzed using IPLab Gel software and gel documentation system (Lightools Research, Encinitas, CA, USA).

\section{Molecular Models}

Molecular models were constructed in Insight II (Accelrys, San Diego, CA, USA). The DNA builder was used to extend the DNA present in the 3-D structure of the $\mathrm{M} \bullet \mathrm{HhaI}$ protein-DNA complex (14) obtained from the Protein Data Bank (PDB) (15) as PDB ID: $1 \mathrm{MTH}$. The arms were linked at the center to form a model of the Y-junction having an accurate sequence. The Assisted Model Building with Energy Refinement (AMBER) molecular force field was used to minimize the structure while constraining the DNA and protein structures in the arms beyond the $10 \mathrm{bp}$ at the center of the junction. The energies of the resulting structures were minimized with respect to molecular mechanics until the root mean square (RMS) force was $<0.1(\mathrm{kcal} / \mathrm{mol}) / \AA$. Following molecular mechanics, each structure was further minimized with 1000 steps of simple dynamics. The structures for the bound and unbound DNA Y-junctions have not been studied by crystallography or by nuclear magnetic resonance (NMR). Thus, it is not clear that the molecular models represent actual conformations for these molecules in solution. Nevertheless, they provide important computer-aided design tools that help to establish the permissible spatial relationships for protein placement on DNA scaffolds $(10,13)$. This allows the investigator to design structures with the highest prospects of success. Moreover, the availability of the 3-D model allows one to study the symmetry of the nanostructure under consideration. As noted below, we have found this useful in estimating the number of electrophoretic forms expected for a given structure. 


\section{RESULTS AND DISCUSSION}

A comparison of the resolution achieved with EMSA and microfluidics is presented in Figure 1. In this experiment, the test oligodeoxynucleotide was a linear duplex with three binding sites for $\mathrm{M} \bullet H h a \mathrm{I}$. The protein/DNA concentration ratio in this experiment permits the visualization of each level of occupancy for the protein-DNA complexes. Thus, free DNA and each of the three levels of occupancy (single, double, and triple) are resolved by both the gel and the microfluidics chip.

The molecular length of the duplex 60-mer is scored accurately by both methods, but neither method registers an expected molecular weight for the protein-DNA complexes. Both methods register unique mobilities for the three complexes. For each system, the observed mobility changes suggest an approximately constant increment corresponding to the addition of a single protein moiety to the complex. Clearly, both systems separate based on structural and surface properties associated with each complex, yet the two systems sense these properties somewhat differently.

Importantly, the virtual gel display produced by the microfluidics system exhibits much higher resolution than the standard $11-\mathrm{cm}$ gradient polyacrylamide gel used for comparison. In particular, it appears to be capable of resolving two forms of the doubly occupied complex. In this system, two forms corresponding to doubly occupied duplexes are observed with mobilities corresponding to that of 310- and 330-bp duplex DNAs. Although the data do not permit an exact assignment, two of the doubly occupied forms are similar to one another such that the central site is occupied and the second protein moiety occupies a site at either end of the duplex. In the third possible form, the two sites at either end of the molecule are both occupied. Thus, based on the symmetry of the molecular models of the system (13), it is reasonable to suggest that one of the two resolved forms corresponds to the mixture of the two forms carrying a protein bound to the central site, while the other form corresponds to the form with proteins bound only at the ends of the molecule.
Methyltransferase-DNA complexes are long lived $(14,16)$, and those targeting $5 \mathrm{FdC}$ can exhibit negligible dissociation (10). Thus, it is unlikely that the differences in resolution observed between the microfluidics and polyacrylamide gel systems are due to dissociation during the separation. It seems more likely to be due to smearing effects associated with low levels of input DNA (17). Moreover, given the adaptability of polyacrylamide gel electrophoresis systems, it is clear that polyacrylamide gels could be developed to separate these forms with a resolution comparable to that of the microfluidics system. However, the real value of the microfluidics approach described here lies in its capacity to achieve high resolution coupled with the overall speed of the analysis, the use of nonradioactive materials, and the requirement for small sample size. This is further demonstrated by the data presented in Figure 2. In this case, a Y-junction recombination intermediate carrying recognition sites for $\mathrm{M} \bullet H h a \mathrm{I}$ at the midpoint of each arm was constructed and decorated with $\mathrm{M} \bullet H h a \mathrm{I}$. The steps in the self-assembly process associated with this supramolecular aggregate are represented schematically in Figure 2A. Figure 2B displays molecular models of the three possible protein-DNA complexes.

Microfluidics separation of the intermediates that can form during the assembly of the supramolecular aggregate is presented in Figure 2C. Since any two single strands have homology extending only over one arm of the junction, when two strands are annealed, they form a partial or frayed duplex. Three such frayed duplexes are possible, and each was observed. The single strands and frayed duplexes were resolved from one another as classes in the microfluidics system. Single strands could be resolved from one another presumably as single-strand conformers, however, the different frayed duplexes were not well separated. When all three strands were annealed, the Y-junction formed spontaneously and uniquely. It was completely resolved from the single strands and frayed duplexes as a lower mobility form. When the annealed Y-junction was mixed with $\mathrm{M} \cdot H h a \mathrm{I}$ protein, mobility-shift products corresponding to each of the 
three possible protein-DNA complexes were observed (Figure 2C). In this case, the symmetry of the Y-junction appears to confine the three levels of occupancy to three electrophoretic forms.

We also investigated the effect of varying the concentration of $\mathrm{M} \bullet H h a \mathrm{I}$ protein at three input concentrations of the Y-junction DNA. The results are presented in Figure 2D. Qualitatively, the results are as expected. That is to say, as the protein/DNA ratio is in- creased, the equilibrium is driven to higher occupancy forms. However, we were unable to use the quantification of the results determined by the Bioanalyzer 2100 to compute reproducible intrinsic binding constants using

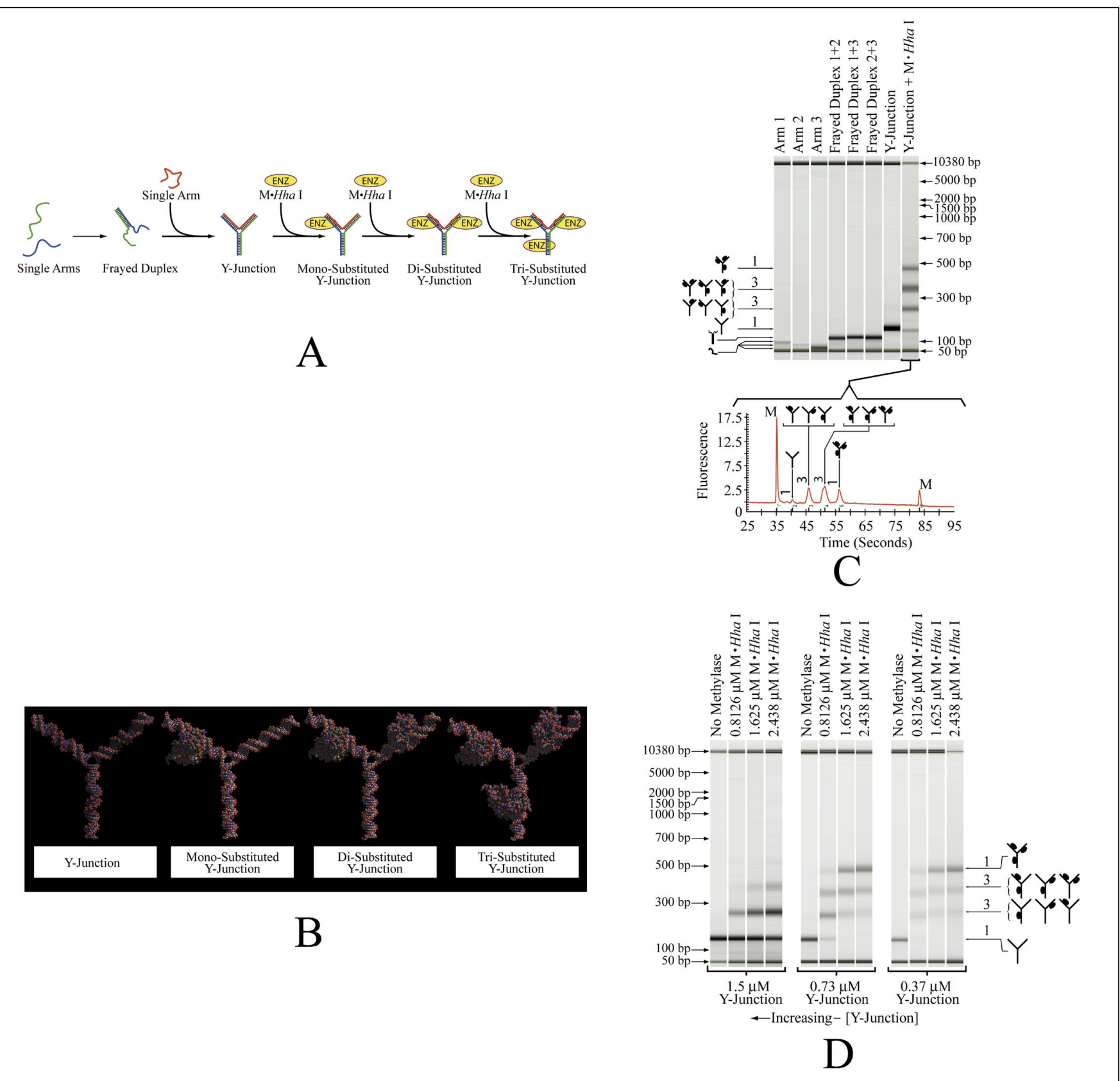

Figure 2. Decorating a recombination intermediate: triskelions for nanotechnological applications. (A) The expected intermediates in the assembly of the Y-junction and its subsequent decoration with methylase $H h a \mathrm{I}(\mathrm{M} \bullet H h a \mathrm{I})$. Single-stranded oligodeoxynucleotides assemble first to a frayed duplex and, upon addition of the third strand, into a Y-junction. Addition of $\mathrm{M} \bullet H h a \mathrm{I}$ produces mono-, di-, and tri-substituted Y-junctions. (B) Molecular models of the unsubstituted, mono-, di-, and tri-substituted Y-junction. Unlike the linearly arranged system described in Figure 1, the symmetry of the Y-junction makes any singly occupied junction equivalent to any other singly occupied junction and any doubly occupied junction equivalent to any other doubly occupied junction. This confines each level of occupancy to a single electrophoretic form. (C) Virtual gel and area scan, detecting each of the intermediates in the assembly of the decorated Y-junction. (D) Substitution level modulation using variation in component concentration levels. At low concentrations of Y-junction DNA (right), increasing the input concentration of $\mathrm{M} \bullet \mathrm{HhaI}$ protein drives the product to full occupancy. At high concentrations of Y-junction DNA (left), mono- and di-substituted forms dominate the product over the same range of input $\mathrm{M} \bullet H h a \mathrm{I}$ protein concentrations. 
standard methods $(4,18)$. Apparently, the bound protein reduces the precision of the proprietary sample detection system used by the Bioanalyzer 2100 . This is perhaps not unexpected since the system has been optimized for the detection of DNA.

In an additional test of the appli- cability of the microfluidics system to mobility shift analysis, we asked whether or not the system could detect mobility shift by a partially purified DNA-binding protein. For this purpose, we used a partially purified preparation of the $\mathrm{M} \bullet E c o$ RII-NLS fusion protein, in conjunction with a Y-junction that contained two binding sites for $\mathrm{M} \bullet H h a \mathrm{I}$ and a single site for the $\mathrm{M} \cdot$ EcoRII-NLS fusion protein. Representative results of this experiment are presented in Figure 3. As expected from the binding site configuration in this Y-junction, a single copy of the M•EcoRIINLS fusion protein gives a mobility shift that is slightly stronger (lower in mobility) than is produced by a single bound copy of $\mathrm{M} \bullet$ HhaI. This observation is consistent with the larger molecular weight of $\mathrm{M} \bullet E c o$ RII (57.5 kDa) compared to that of $\mathrm{M} \bullet H h a \mathrm{I}(39.5$ $\mathrm{kDa}$ ). Moreover, when $\mathrm{M} \bullet H h a \mathrm{I}$ is mixed with the Y-junction in isolation, only two forms are observed as expected from the presence of only two binding sites for this protein. When all three components of the system (Y-junction, $\mathrm{M} \bullet H h a \mathrm{I}$ and $\mathrm{M} \bullet$ EcoRII-NLS fusion) were incubated together, all three of the expected mobility-shifted forms were observed.

As noted in the introduction, the construction of biosensors and the development of complex programmable devices with bionanotechnology $(8,10,13,19)$ will require the development of several enabling
Figure 3. Placing a fusion protein at a preselected site in decorated recombination intermediate. By using a Y-junction with two recognition sites, one for methylase $\mathrm{Hha \textrm {I }}(\mathrm{M} \bullet \mathrm{HhaI})$ and one for methylase EcoRII (M•EcoRII) on preselected arms of the Y-junction, the system can address two components to the recombination intermediate. In this example, a nuclear localization signal (NLS) has been fused to $\mathrm{M} \bullet E c o$ RII to demonstrate the selective positioning of a fusion protein. 
tools. In particular, preparative separation of systems like the decorated recombination intermediates described here will be required. Preparative polyacrylamide gel electrophoresis, FPLC, and density gradient centrifugation are candidate preparative methods that should be useful for this purpose. On the other hand, the microfluidics system described here can be used in cataloging occupancy and solution symmetry of nanostructures similar to those studied here. Moreover, interesting protein-protein interaction studies using the microfluidics system could be contemplated if DNA-protein complexes or nanostructures can be shown to exhibit supershifts in the presence of antibodies or candidate partnerproteins.

We conclude that the Bioanalyzer 2100 and the DNA LabChip system, although designed for accurate and rapid sizing of DNA fragments, can also be effectively applied to the rapid detection of protein-DNA mobility shifts of interest in molecular biology, as well as the complex supramolecular aggregates now emerging from bionanoscience $(7,8,10,13,19-27)$.

\section{ACKNOWLEDGMENTS}

This work was supported by grant no. 0395 from the Smokeless Tobacco Research Council, USA, by the National Institutes of Health (NIH) grant no. 1G08LM06722-01A1 from the National Library of Medicine, by NIH Grant CA 91234 from the National Cancer Institute, and by a grant from the Ella Fitzgerald Foundation. We thank David Baker and John Wendel for technical support.

\section{REFERENCES}

1.Bain, D.L. and G.K. Ackers. 1998. A quantitative cryogenic gel-shift technique for analysis of protein-DNA binding. Anal. Biochem. 258:240-245.

2.Brenowitz, M., D.F. Senear, and G.K. Ackers. 1989. Flanking DNA-sequences contribute to the specific binding of cI-repressor and OR1. Nucleic Acids Res. 17:3747-3755.

3.Kerr, L.D. 1995. Electrophoretic mobility shift assay. Methods Enzymol. 254:619-632.

4.Senear, D.F. and M. Brenowitz. 1991. Determination of binding constants for cooperative site-specific protein-DNA interactions using the gel mobility-shift assay. J. Biol. Chem. 266:13661-13671.

5.Senear, D.F., D.D. Dalma-Weiszhausz, and M. Brenowitz. 1993. Effects of anomalous migration and DNA to protein ratios on resolution of equilibrium constants from gel mobility-shift assays. Electrophoresis 14: 704-712

6.Onizuka, T., S. Endo, M. Hirano, S. Kanai, and H. Akiyama. 2002. Design of a fluorescent electrophoretic mobility shift assay improved for the quantitative and multiple analysis of protein-DNA complexes. Biosci. Biotechnol. Biochem. 66:2732-2734.

7.Smith, S.S. 2002. Designs for the self-assembly of open and closed macromolecular structures and a molecular switch using DNA methyltransferase to order proteins on nucleic acid scaffolds. Nanotechnology 13:413-419.

8.Smith, S.S. 2003. Nucleoprotein Assemblies, X, p. 1-10. In H.S. Nalwa (Ed.), The Encyclopedia of Nanoscience and Nanotechnology. American Scientific Publishers, Stevenson Ranch, CA.

9.Gibson, T.J. and A.I. Lamond. 1990. Metabolic complexity in the RNA world and implications for the origin of protein synthesis. J. Mol. Evol. 30:7-15.

10.Smith, S.S., L. Niu, D.J. Baker, J.A. Wendel, S.E. Kane, and D.S. Joy. 1997. Nucleoprotein-based nanoscale assembly. Proc. Natl. Acad. Sci. USA 94:2162-2167.

11.Buryanov, Ya.I., I.G. Bogdarina, and A.A. Bayev. 1978. Site specificity and chromatographic properties of E. coli $\mathrm{K} 12$ and EcoRIIDNA-cytosine methylases. FEBS Lett. 88: 251-254.

12.Kosykh, V.G., A.S. Solonin, Ya.I. Buryanov, and A.A. Bayev. 1981. Overproduction of the EcoRII endonuclease and methylase by Escherichia coli strains carrying recombinant plasmids constructed in vitro. Biochim. Biophys. Acta 655:102-106.

13.Smith, S.S. 2001. A self-assembling nanoscale camshaft: implications for nanoscale materials and devices constructed from proteins and nucleic acids. Nano Lett. 1:51-56.

14.Klimasauskas, S., S. Kumar, R.J. Roberts, and X. Cheng. 1994. HhaI methyltransferase flips its target base out of the DNA helix. Cell 76:357-369.

15.Berman, H.M., J. Westbrook, Z. Feng, G. Gilliland, T.N. Bhat, H. Weissig, I.N. Shindyalov, and P.E. Bourne. 2000. The Protein Data Bank. Nucleic Acids Res. 28:235-242.

16.Verdine, G.L. 1994. The flip side of DNA methylation. Cell 76:197-200.

17.Smith, S.S., T.E. Gilroy, and F.A. Ferrari. 1983. The influence of agarose-DNA affinity on the electrophoretic separation of DNA fragments in agarose gels. Anal. Biochem. 128:138-151.

18.Shea, M.A. and G.K. Ackers. 1985. The OR control system of bacteriophage lambda. A physical-chemical model for gene regulation. J. Mol. Biol. 181:211-230.

19.Smith, S.S. 1995. Nucleoprotein-based nanoscale fabrication. Biological and Biomedical Science and Technology Division ONR 34196-3:161-162.

20.Montemagno, C.D. 2001. Nanomachines: a roadmap for realizing the vision. J. Nanopart.
Res. 3:1-3.

21.Niemeyer, C.M. 2000. Self-assembled nanostructures based on DNA: towards the development of nanobiotechnology. Curr. Opin. Cell Biol. 4:609-618.

22.Niemeyer, C.M., M. Adler, B. Pignataro, S. Lenhert, S. Gao, L. Chi, H. Fuchs, and D. Blohm. 1999. Self-assembly of DNAstreptavidin nanostructures and their use as reagents in immuno-PCR. Nucleic Acids Res. 27:4553-4561.

23.Niemeyer, C.M., L. Boldt, B. Ceyhan, and D. Blohm. 1999. DNA-directed immobilization: efficient, reversible, and site-selective surface binding of proteins by means of covalent DNA-streptavidin conjugates. Anal. Biochem. 268:54-63.

24.Niemeyer, C.M., J. Koehler, and C. Wuerdemann. 2002. DNA-directed assembly of bienzymic complexes from in vivo biotinylated NAD(P)H:FMN oxidoreductase and luciferase. Chembiochem 3:242-245.

25.Niemeyer, C.M., T. Sano, C.L. Smith, and C.R. Cantor. 1994. Oligonucleotide-directed self-assembly of proteins: semisynthetic DNA - streptavidin hybrid molecules as connectors for the generation of macroscopic arrays and the construction of supramolecular bioconjugates. Nucleic Acids Res. 22:55305539.

26.Seeman, N.C. 1998. DNA nanotechnology: novel DNA constructions. Annu. Rev. Biophys. Biomol. Struct. 27:225-248.

27.Yang, X., L.A. Wenzler, J. Qi, X. Li, and N.C. Seeman. 1998. Ligation of DNA triangles containing double crossover molecules. J. Am. Chem. Soc. 120:9779-9786.

Received 18 April 2003; accepted 6 June 2003.

Address correspondence to:

Jarrod Clark

Kaplan Clinical Research Laboratory

City of Hope Medical Center

1500 E. Duarte Road

Duarte, CA 91010, USA

e-mail: jclark@coh.org 